\title{
El papel de la reforma del funcionariado en la lucha contra la corrupción *
}

\section{David Shand ** y Safdar Sohail ***}

\section{Introducción}

En muchos países en vías de desarrollo, la administración pública ha sido una institución cerrada y jerárquica, a menudo insensible a las necesidades de los ciudadan os y tendente a abusar de su autoridad. Los distintos effuerzos de reforma han tratado de abordar estos problemas. Las tradicionales reformas del funcionariado se han centrado en la organización y estructuras del funcionariado; temas de gestión del personal, tales como las pensiones, sistemas de clasificación, reclutamiento y formación; y el desarrollo de marcos éticos. Es difícil encontrar indicadores fiables de cuál ha sido su impacto en la corrupción, principalmente porque estas reformas no tuvieron expresamente un objetivo anticorrupción. Pero los datos de que se dispone sugieren que han tenido un modesto impacto en frenar la oleada de corrupción.

Más recientemente, las dos aproximaciones más importantes a la reforma del funcionariado han tratado de forma más directa de reducir la corrupción. La primera aproximación ha tratado de reducir el número de funcionarios para mejorar las condiciones degradantes en que los funcionarios prestan sus servicios, que se percibían como incentivadoras de la corrupción. La segunda se centró en las iniciativas de gestión del desempeño y en la construcción de la capacidad institucional. Es difícil medir el impacto de estas reformas sobre la corrupción, pero la bibliografía disponible sugiere que su impacto también ha sido modesto. Sin embargo, la discusión se ha centrado más en que las reformas tengan un objetivo más expreso de controlar la corrupción.

Este capitulo explora las conexiones entre tales reformas y la lucha contra la corrupción - para ayudar a diseñar las reformas que combatirán de forma mas eficaz la corrupción y también mejorarán el desempeño del funcionariado- . Pero también se debe prestar atención a la agenda cada vez más amplia de la reforma de la gestión pública que se está llevando a cabo en muchos páses, que incluye la descentralización, la privatización, el desarrollo de un gobierno orientado a los ciudadanos y las técnicas de gestión del rendimiento. Estas reformas recientes ofrecen una experiencia valiosa a la hora de diseñar nuevas iniciativas para luchar contra la corrupción en el funcionariado. Este artículo, por tanto, adopta un punto de vista amplio del término «reforma del funcionariado", al incluir las reformas de los sistemas de gestión pública diseñados para mejorar la gestión del funcionariado.

\section{Cambios en las estructuras del funcionariado}

En un sistema de gobierno que funcione bien, el rol del gobierno y su estructura organizacional son mutuamente compatibles. Si cambia el rol del gobierno, las estructuras también deben cambiar. Las tres áreas más importantes de cambio han tenido 0 están teniendo lugar: descentralización hacia los gobiernos subnacionales, creación de organismos autónomos y privatización.

\section{D escentralización}

Se puede llevar a cabo la descentralización hacia niveles inferiores del gobierno por varias razones: para incrementar la eficiencia y responsabilidad en la prestación de servicios, responder a presiones políticas y regionales en favor de un gobierno más 
descentralizado y luchar contra la corrupción. Muchos estudios recientes han demostrado la susceptibilidad de las instituciones centralizadas hacia mayores niveles de corrupción. La concentración del poder en unas pocas manos 0 en algunas instituciones crea monopolios y falta de transparencia lo que, a su vez, incre menta las probabilidades de corrupción.

M uchos países han iniciado la descentralización en los últimos años para vencer estos problemas, presuponiendo que la descentralización podría proporcionar el beneficio de la proximidad, haría posiblequela sociedad civil exigiese una mejor gestión y mejoraría la transparencia. De esta forma, el gobierno central podría jugar un papel regulador en la prestación del servicio. El gobierno central podría establecer estándares, ofrecer apoyo y aceptar la responsabilidad global ante el electorado, pero los gobiernos locales prestarían los servicios públicos.

Es demasiado pronto para hacer una evaluación concluyente de esta descentralización, pero la bibliografía sobre el tema sugiere unas cuantas lecciones:

1. La calidad de la prestación descentralizada de los servicios puede deteriorarse, al menos, en un principio.

2. La duplicación de la actividad entre los gobiernos central y local y la apropiación indebida de fondos por parte de los funcionarios locales son riesgos potenciales.

La administración en general y el reclutamiento en particular pueden ser más susceptibles de interferencias políticas en los niveles descentralizados. Allí donde las regiones se diferencian en función de líneas étnicas, es significativa la amenaza de clientelismo étnico.

La diferencia de capacidad entre el poder central y los niveles locales, o entre los diferentes niveles locales, puede ser lo suficientemente grande como para sostener una descentralización significativa. En un país escaso de recursos humanos, la descentralización puede empeorar la situación, al reducir la equidad y eficiencia en la prestación del servicio.

Para evitar estos problemas, la literatura señala ciertas precondiciones que permiten una prestación de servicios mejor y menos corrupta en el ámbito de la descentralización. Algunas de estas condiciones son:

3. Se debería revisar cuidadosamente la capacidad administrativa del gobierno central y de las regiones. Por ejemplo, puede ser necesario transferir funcionarios ya formados del nivel central a las regiones - lo cual puede ser difícil de conseguir.

4. Ladescentralización deberíair acompañadadela construcción de una capacidad anticorrupción en el nivel local.

5. Se deberían desarrollar los controles políticos y administrativos adecuados en el punto de prestación del servicio, para garantizar la responsabilidad y la eficiencia.

6. Se debería desarrollar el sentimiento de propiedad por parte de la sociedad en todo este proceso.

\section{Creación de organismos autónomos}

En algunos países, las funciones específicas del gobierno se han extraído del núcleo de la administración pública y se han transferido de los ministeriosa organizaciones separadas, quefuncionan con más autonomía de gestión. Esta autonomía puede comportar una mayor libertad respecto de las reglas del funcionariado (que tienen que ver con la cantidad de personal, salarios y otras condiciones del servicio) y una mayor autonomía presupuestaria de la que disfrutan los ministerios. En la práctica, el nivel de autonomía puede diferir entre países y entre organizaciones dentro del mismo país.

Las razones para crear organismos autónomos varían, pero incluyen la dificultad ya observada de operar un determinado servicio según las reglas del funcionariado que se aplican a los ministerios y la necesidad de separar la intervención organizacional de la política o ministerial en sus decisiones de funcionamiento. Por ejemplo, muchos países han creado organismos autónomos para recaudar impuestos y derechos de aduana con el fin de reclutar personal externo y pagarles mejor, además de distanciarse ellos mismos de la intervención ministerial. En algunos casos, los benefactores internacionales pueden fomentar o exigir una propuesta de enclave para prestar determinados servicios 0 actividades. A cambio de la autonomía de gestión adicional, a los organismos se les ha exigido, al menos en teoría, ser más responsables y cumplir los objetivos de mejorar la gestión.

Pero también parece que ha habido algunas razones inapropiadas para su creación. La capacidad de puentear las limitaciones salariales del funcionariado puede emplearse más para el be neficio personal de la dirección y del staff, que para mejorar los servicios al público. Las organizaciones que tienen sus propias fuentes de ingresos también pueden ser una gallina de los huevos de oro. D e igual manera, los clientes pueden hacerse con el control de las organizaciones, p.e. los productores pueden controlar las juntas de producción agrícolas y pueden usar los fondos más para su propio beneficio que para el consumo público.

$H$ ay pruebas de que los organismos autónomos desencadenan más corrupción - que va desde el nepotismo hasta salarios excesivos y la total apropiación indebida de fondos-. Esto refleja el hecho de que, en muchos casos, se concedió una autonomía de gestión adicional, sin quese exigiera la consiguiente responsabilidad y transparencia adicional por la gestión. Se usó inapropiadamente el poder discrecional cada vez mayor que se dio a la dirección.

Los programas de reforma del funcionariado que ahora se lle van a cabo en algunos países reconocen la necesidad de exigir responsabilidad a los organismos autónomos. Los programas que se pusieron en práctica (como la tran sparencia en la planificación corporativa y el establecimiento de objetivos de gestión) mejoraron el tema de la responsabilidad. 


\section{Privatización}

M uchos países en vías de desarrollo han comenzado a privatizar empresas propiedad del Estado en las pasadas dos décadas para abordar la ineficiencia y la corrupción en el sector paraestatal. Pero esta reforma está lejos de completarse y plantea sus propios riesgos de corrupción. Son frecuentes los ejemplos de privatizaciones dudosas y apresuradas. Para lograr los beneficios que se pretenden, los esfuerzos de reforma deberían centrarse en las siguientes áreas:

7. Extender las reformas. Puede haber ciertos sectores $u$ organizaciones que todavía no se han destinado a ser privatizados, debido al interés de algunos en mantener el monopolio.

8. Transparencia del proceso de privatización. Sólo una venta transparente puede obtener los mejores resultados. En muchos países, el proceso de privatización se asocia con el aumento de la corrupción a gran escala y se diceque se ha desarrollado un nexo entre los políticos, los funcionarios y sus hombres de negocios favoritos.

9. Proteger los intereses de los consumidores. La privatización no debería tener como resultado una pérdida de los legítimos intereses de los ciudadanos. En muchos casos, no se pusieron en práctica las salvaguardas y regulaciones debidas para proteger los intereses de los consumidores sobre los monopolios privatizados, como la electricidad y el agua. Es más difícil imponerlos más tarde. El coste social del monopolio privado no regulado puede ser alto si los servicios clave llegan a ser demasiado caros para los ciudadanos normales y corrientes.

\section{M ejorando las condiciones del funcionariado}

Las condiciones de trabajo de un empleado pueden afectar significativamente a su comportamiento. Se admite con carácter general que la naturaleza de las condiciones de trabajo tiene un impacto directo en la corrupción, de tal forma que cuanto mejores sean las condiciones de trabajo, es menos probable que los funcionarios sean corruptos.

Pero mejorar las condiciones del funcionariado, especialmente los salarios, no es una condición suficiente para erradicar la corrupción. No se debería exagerar ni subestimar su relevancia en las estrategias anticorrupción. El debate se centra en dos te mas principales: (i) la idoneidad de salarios, pensiones y otros beneficios salariales en dinero o no; $y$ (ii) las condiciones genera- les del trabajo y de la carrera profesional. El segundo tema se trata en la siguiente sección y versa sobre los cambios en la gestión del personal. La posibilidad de una aproximación más selectiva para mejorar los salarios y demás condiciones también se examina en esa sección.

\section{Remuneración adecuada}

Se considera que la corrupción en pequeña escala basada en la necesidad es más una cuestión de «compensación justa» que una cuestión moral y muchos creen que unos salarios adecuados la harán fracasar. Hay muchos estudios sobre este tema, entre los que se incluye un importante estudio del FM I de 1997. Este estudio reconoce la importancia de una adecuada remuneración para garantizar un funcionariado honesto, pero discrepa de su magnitud e identifica otros factores igualmente importantes, tales como el imperio de la ley, la calidad de la burocracia y la eficacia de los controles internos y externos. La importancia relativa de incrementar la remuneración hasta alcanzar niveles ade cuados sigue siendo una cuestión abierta. De hecho, el tema del salario adecuado es muy complejo.

Si no recibe un salario adecuado, el funcionario podría compensarlo con el pluriempleo, con la dependencia de los recursos de la familia 0 aceptando/solicitando sobornos - si el riesgo de ser descubierto se considera insignificante o si la persona no considera poco ético aceptar sobornos-. En este sentido, los empleados se pueden distribuir a lo largo de un espectro en el que en un extremo está la gente bien pagada, con una aversión moral hacia la corrupción y que tienen miedo de ser descubiertos y en el otro extremo, los mal pagados, sin escrúpulos, que no temen ser descubiertos. Entre medias, habrá una gama de situaciones que podrían incluir:

- Personas mal pagadas con una aversión moral hacia la corrupción, que complementan sus ingresos por medios privados legales.

- Personas mal pagadas, moralmente ambivalentes, pero que temen ser descubiertas y que complementan sus ingresos por medios privados legales.

- Personas mal pagadas, moralmente ambivalentes, que aceptan sobornos.

La valoración de los que elaboran las políticas públicas sobre dóndeestán en líneas generales dentro de esteespectro sus funcionarios públicos informa de la dirección de la política de compensación del gobierno. En la mayoría de los países de la 0 CDE, los salarios mínimos definen explícita o implícitamente las necesidades sociales mínimas. Al establecerlo, los gobiernos se proponen empujar a la gente hacia la parte más alta del espectro, como se ha señalado anteriormente. En un país en vías de desarrollo, la distribución delosempleadosalo largo del espectro es más compleja- 
de ahí también la tarea de desarrollar una política adecuadaCada país tiene su propia situación concreta, pero el papel de las condiciones del funcionariado en el fomento de la corrupción se corresponde generalmente con este model o de distribución.

Por tanto, la investigación sugiere que la disposición del empleado haciala corrupción no depende sólo del salario; antes bien, también son relevantes las actitudes éticas, el temor de ser descubierto y la presencia 0 ausencia de lo que se denomina «calidad de la burocracia» (satisfacción de los funcionarios públicos con su carrera y sus expectativas). Por esta razón, ha resultado difícil determinar un «salario incentivador» para reducir la corrupción. Parece que la aproximación más fructífera es considerar el caso específico de cada país y atender, al mismo tiempo, a los aspectos de no compensación de las condiciones del funcionariado - que se examinan en la siguiente sección de este documento-.

La búsqueda de criterios que definan el salario adecuado ha llegado a ser más bien partidista, entre aquéllos que están a favor de usar el sector privado como un punto de referencia y aquéllos que resaltan las dificultades de comparar los dos sectores en un país con un desempleo muy al to y un sector privado débil. Dadas las restricciones presupuestarias reales, una mejora gradual y coherente en los salarios del funcionariado parece ser la forma más realista de alcanzar un salario que les permita vivir.

La compresión de los salarios es otro aspecto de lo que se considera un salario adecuado. En África, por ejemplo, el diferencial entre los funcionarios mejor y peor pagados iba de 20:1 a 30:1 antes de la independencia. Este diferencial se redujo más tarde en aras de la equidad y en algunos países ha descendido hasta 6:1 hoy en día. Para algunos observadores, los bajos salarios de los funcionarios senior indican que el gobierno no setoma en serio la corrupción. Incluso se ha observado que los funcionarios senior toman más de lo que necesitan mediante la corrupción, de tal forma que unos salarios más altos no contrarrestarían necesariamente su avaricia. Además, muchos temen que subir de forma selectiva los salarios de los funcionarios senior desmoralizaría a la mayoría de los funcionarios públicos y sostienen que primero, un gobierno debería mejorar el nivel general de los salariosy después, restablecer gradualmente un diferencial salarial razonable.

Una vez que se han definido el salario adecuado y el diferencial salarial, surge la cuestión de la viabilidad financiera. Las anteriores reformas del funcionariado han intentado crear más recursos para los incrementos y la descompresión salarial haciendo recortes de plantilla, reducciones, paquetes de despidos, reduciendo el número de ministerios y eliminando los trabajadores fantasma. Por tanto, manteniendo más o menos igual la partida total de salarios, se esperaba que el gobierno pudiera pagar más a losfuncionarios que quedaban. En al gunos casos, esto ha funcionado, como en U ganda, pero en otros casos, no, como en M alawi, donde los ministerios no devolvieron los recursos al erario público después de las reducciones.
Se han intentado acometer otras propuestas de una remuneración adecuada con menos impacto. En muchos países se ha intentado la cuantificación de beneficios complementarios, tales como dar una casa o un vehículo, pero sus resultados no están claros. Pagar en función del rendimiento también se ha puesto en práctica, pero los resultados no son significativos en la mayoría de los casos. La mejor aproximación parece ser una buena gestión de los recursos disponibles y poner en práctica una política de salarios adecuada a largo plazo.

\section{M ejorar de forma selectiva las condiciones en el núcleo del fun- cionariado}

La literatura sobre la reforma del funcionariado sugiere que un desarrollo sistemático de la inversión en un funcionariado central o nuclear ayuda a reducir la corrupción. U na aproximación para construir un funcionariado nuclear se hace a través del establecimiento de objetivos en los servicios claves para mejorar sus condiciones. Si se van a establecer gradual mente salarios ade cuados para reducir la corrupción, se deben fijar objetivos para los servicios clave, tales como los vinculados al imperio de la ley, la administración general, la administración financiera y la elaboración de la política económica primero, para aumentar los salarios, además de para conseguir mayores estándares (recompensas y sanciones) de comportamiento.

Al mismo tiempo, se pueden conceder las funciones de exigir responsabilidad y control del funcionariado a las instituciones centrales, que lideran el resto de los servicios prestados por los funcionarios. Pueden tener protección estatutaria para soportar mejor la presión política y se puede dar una alta prioridad a su reclutamiento, formación y condiciones de trabajo (p.e. Comisiones de Funcionariado, O ficina de Auditoría, Autoridad de Ingresos). Pero la creación de tales islas de privilegio puede crear dificultades en el resto del funcionariado, al menos al principio.

\section{Cambios en la gestión del personal}

Los objetivos de un sistema de gestión de personal son crear una estructura que permita sacar lo mejor del contrato de trabajo, tanto para el gobierno como para el empleado. Generalmente, un funcionario es un empleado permanente y se espera que actúe de forma coherente con el contrato y el rol de funcionario público. A cambio, el empleado espera ciertos beneficios, tales como seguridad de permanencia, tareas relevantes y prometedoras, un salario fijo de forma predecible, incrementos salariales a 
tiempo, oportunidades de promoción, pensiones y otros beneficios directos o indirectos. Para institucionalizar esta mutualidad, los gobiernos crean varias instituciones tales como los M inisterios o Comisiones de Funcionariado y los departamentos de personal de los ministerios. Se desarrollan normas y reglamentos y prácticas de recompensa y sanciones. Todas esas reglas, en tanto en cuanto encumbran la importancia de la eficiencia, declaran que el uso del cargo público en beneficio personal propio supone un incumplimiento de las reglas y establecen mecanismos específicos para tratar dichos incumplimientos.

Por tanto, un país con un funcionariado corrupto e ineficiente debería examinar el funcionamiento de su sistema de gestión del personal, averiguar la natural eza de todos los fallos y descubrir métodos para reformarlo. La bibliografía relevante en este sentido identifica tres grandesáreas en la gestión del personal, que podrían correlacionarse directa o indirectamente con la corrupción:

10. Carencia o fracaso en la implementación de controles anticorrupción internos claros

11. Insatisfacción de los funcionarios con su carrera 0 expectativas

12. Interferencia política o deotro tipo en el reclutamiento.

\section{Controles internos contra la corrupción}

Reglas inadecuadas o una debilidad en su implementación favorecerán a la corrupción. Gran parte de la corrupción en pe queña escala, en concreto, podría controlarse mejorando las re glas y asegurándose de que los funcionarios senior llevan a cabo una correcta implementación de las mismas. Una estrategia anticorrupción eficaz dentro de una organización tiene que ver con los siguientes componentes: (i) recibir y procesar reclamaciones; (ii) llevar a cabo una encuesta; (iii) tomar una decisión o emitir una opinión; (iv) procedimientos de apelación; y (v) posible continuación en un tribunal penal.

0 tro aspecto de la ineficacia de los controles internos contrala corrupción es la disociación en la mayoría de los casos entrelos controlesinternosy los controles externos. La auditoría es un buen ejemplo. L os departamentos del gobierno son reticentesa verseenvueltos en una auditoría externa, incluso cuando saben que tienen serios problemas. En la mayoría de los casos, las encuestas llevadas a cabo dentro del departamento sobre quejas o sospechas no conduce a una auditoría externa especial sistemática o a su persecución. Para salvar la reputación de la organización, las encuestas se manejan internamente y no sellevan a cabo para sus fines lógicos. La misma mentalidad prevaleceen lo que respecta aorganismosanticorrupción. La sociedad civil, queseesperajuegue un papel central en el control de la corrupción, apenas tiene influencia en este escenario. Existeuna necesidad de revisar la conexión entre la 0 ficina del Auditor G eneral, los Comités de Contabilidad Pública, los organismosanticorrupción y los departamentos del gobierno en la ejecución de controles internos.

\section{Insatisfacción con la carrera y expectativas}

Un sistemadegestión del personal quefuncionadeficientemente crea un funcionariado desmotivado, que oculta los motivos de quejay con una pobreimagen desí mismo. Lossistemas inadecuados para satisfacer las quejas, las promociones injustas, las políticas opacas 0 arbitrarias de recortes, unas estructuras y niveles caóticos de los funcionarios públicos y una gestión deficiente de la información son al gunas de las causas de la pérdida de perspectiva moral. M ientras que la literatura sobre esta relación causal es bastantegeneral, losdatossugieren que existeuna mayor probabilidad decorrupción por partede los funcionarios públicosqueestán insatisfechos con sus trabajos o no están seguros de su futuro.

Un estudio del comportamiento de los funcionarios públicos sobre los temas de personal en Europa del Este y C entral sugiere que los empleados que tenían miedo de ser despedidos tendian a ser más corruptos. En G hana, el destino de miles de funcionarios estuvo sin decidir durante al gunos años, lo que se dijo que había tenido efectos negativos en la moral global del funcionariado público.

Pero lo más insatisfactorio para un funcionario es la necesidad de pagar sobornos para conseguir beneficios tales como salarios, anticipos o una casa para alquilar. Esto puede disminuir su inhibición frente al hecho de llegar a estar involucrado en la corrupción ${ }^{1}$. En tales circunstancias, al gún miembro honesto del staff podría irse y al gunos podrían reducir sus propios estándares éticos, vengándose con el sistema.

I gualmente, se ha descubierto una correlación positiva entre un alto nivel de satisfacción con su carrera y expectativas (a veces denominada «calidad delaburocracia») y unaadministración más eficiente y menos corrupta. M antener la satisfacción del empleado es, por tanto, un área importante en la reforma del funcionariado. Sería un indicador esencial del rendimiento de un sistema de gestión del personal.

\section{Interferencias políticas o de otro tipo en el reclutamiento}

D ados los niveles de desempleo en los países en vías de desarrollo, la gente está dispuesta a pagar a los políticos o funcionarios que controlan el reclutamiento para conseguir un trabajo. Si además de un salario, hay oportunidades de ganar dinero a través de prácticas corruptas, el nivel de pago aumentará. Las consecuencias de esto son claras. Si el reclutamiento se realiza sobre otra base que la del mérito, es difícil evitar la corrupción sistémica. Es muy importante apoyarse en el mérito en el reclutamiento y la promoción para luchar contra la corrupción. La cuestión es tan importante que en al gunos casos la sociedad civil ha demandado la intervención de benefactores internacionales para asegurarse de la selección de gente cualificada para posiciones muy senior. 
En esta área es evidente el importante papel de las Comisiones independientes del Funcionariado. I gualmente, es importante la capacidad de los funcionarios de apelar razonablemente a alguna autoridad de revisión independiente sobre los nombramientos y promociones.

\section{O tras reformas en la gestión del personal}

0 tras reformas para introducir sistemas de gestión del personal más modernos y basados en el rendimiento también pueden tener implicaciones en el nivel de corrupción. Éstas incluyen:

- especificaciones claras de responsabilidades o descripciones de puestos. Si los funcionarios no están trabajando según tareas claramente definidas, se incrementa la posibilidad de actividades irregulares que lleven a la corrupción;

- sistemas estandarizados de clasificación de puestos que garanticen que existen las adecuadas relaciones entre los salarios pagados por los diferentes tipos de trabajo y la equidad de salarios para el mismo tipo de trabajo. La corrupción puede tener lugar al establecer salarios inapropiadamente altos, a menos que haya un sistema estandarizado de clasificación de puestos - y que se use y se aplique este sistema;

- evaluaciones del desempeño regulares de cada miembro de la plantilla. Si se llevan a cabo con profesionalidad y con integridad, tales evaluaciones pueden dejar al descubierto el desempeño deficiente que puede reflejar que dejan de atender sus obligaciones a causa del «pluriempleo» ilegal en el sector privado, o el nombramiento de personas no cualificadas a través de la corrupción o el nepotismo. Pero es importante que tales evaluaciones del rendimiento tengan consecuencias; si los que tienen un bajo desempeño no son cesados o sancionados disciplinariamente, el sistema tendrá poco impacto;

- designacionespor contrato definalización determinada para los funcionarios senior. Las designaciones por contrato pue den centrarse más específicamente en la gestión y desincentivar aśl la corrupción, aunque la falta de permanencia podría animar a adoptar un punto de vista a corto plazo, prestando atención a las cuestiones éticas. Por tanto, queda como cuestión abierta hasta qué punto tienen impacto sobre la corrupción, si es quetienen al guno, las designaciones por contrato.

\section{Desarrollando marcos éticos en el funcionariado}

En cualquier estrategia anticorrupción juega un papel importante el conocer a ciencia cierta cuáles son las indicaciones éticas de la corrupción. M uchos países han desarrollado marcos éticos, que tratan de definir los aspectos éticos del funcionariado. Tales marcos tratan de socializar y educar a los funcionarios públicos más que definir y hacer cumplir solamente las reglas. La aceptación de los valores informales que absuelven a los individuos de la culpabilidad moral es probablemente el escollo más importarte para conseguir el compromiso por parte de los funcionarios públicos para luchar contra la corrupción.

D esarrollar un entorno ético es un proceso lento y puede te ner éxito, sólo como parte de una estrategia holística. La mera retórica sobre los valores éticos, sin acciones concretas, puede ser contraproducente y generar más cinismo. Una vez que se han desarrollado, los códigos éticos de conducta requieren su aplicación.

En muchos países, los marcos éticos abarcan a todos los funcionarios del sector público, no sólo los funcionarios clave. Se ha pensado seriamente en identificar los elementos que pueden actuar como incentivos del buen comportamiento y que desincentivan la corrupción. Los elementos de un marco ético, a veces denominado infraestructura ética, comprenden:

Órganos de coordinación

M arco legal

Mecanismos de responsabilidad

Códigos de conducta

Socialización profesional

Condiciones del funcionariado

Compromiso político

Implicación y escrutinio por parte de la sociedad

La mezcla y ajuste de estos componentes dependerá del sistema cultural y político/administrativo del país y sus iniciativas pasadas en promover un marco ético. Tales infraestructuras éticas son nuevas, incluso en los países del la O CDE y es demasiado pronto para comentar su eficacia. Pero como cualquier otra herramienta, su eficacia depende de si se han entendido y usado coherentemente.

A este respecto, el papel de liderazgo delos órganos de coordinación para promulgar un código ético en el funcionariado es importante. Pueden existir departamentos encargados de la gestión del funcionariado o se puede crear un nuevo departamento. Éstos educan al funcionariado y a la sociedad, les hacen conscientes de las cuestiones éticas y apoyan el desarrollo de las habilidades en el análisis ético.

Los códigos de conducta también son importantes para hacer que el comportamiento de los individuos sea conforme con todas las expectativas éticas. Un código de conducta estándar contiene exigencias en las siguientes áreas:

13. Declaración de valores

14. Declaración de conflictos de interés 
15. Reglas sobre la recepción de regalos

16. Juramentos de honestidad e imparcialidad

17. Cartas de los ciudadanos, que establecen la exigencia de servir a los ciudadanos.

Estos códigos incorporan nuevos valores clave, que resaltan la eficiencia, orientación al cliente y la responsabilidad, junto con los tradicionales valores centrales tales como la honestidad, inte gridad, justicia y el no partidismo. En resumen, significa que el funcionario público debe estar imbuido de una gama más amplia de valores que antes.

\section{D elatar a los corruptos}

$H$ ay varias formas de delatar a los corruptos. Ser testigo de un acto corrupto plantea cuestiones éticas importantes, además de legales. M ientras que los países dan respuestas distintas a estas cuestiones, hay un acuerdo generalizado sobre que el delatar a un corrupto o informar de un acto ilegal es un mecanismo importante para reducir la corrupción y mejorar la prestación de servicios. Los informes pueden venir tanto del funcionario público como del cliente/usuario. Pueden adoptar la forma de negarse a manejar una transacción en donde se sabe que está presente la corrupción, o filtrar información a los medios. Más directamente, puede hacerse informando de los actos ilíticos a los funcionarios de forma confidencial o hacerlo a través de quejas por escrito. Los gobiernos pueden facilitar el delatar a los corruptos incrementando la transparencia en el proceso de toma de decisiones, de tal forma que los funcionarios públicos y los ciudadanos pueden observar e informar los actos ilícitos.

Dado el rol potencialmente importante, la delación ha sido centro de atracción de la reforma en muchos países de la $O C D E$. Se ha informado de una reducción en la corrupción, tras la introducción de las reglas de la delación en Holanda y Austria. Se acepta generalmente que una fuerte presencia de este tipo de informaciones es una indicación de un nivel cada vez más alto de la toma de conciencia y seriedad en la lucha contra la corrupción.

El éxito de un sistema de información depende de muchos factores, que pueden variar de un país a otro. La delación siempre ha sido más problemática para los funcionarios públicos que trabajan dentro de las nociones tradicionales de lealtad a los superiores y solidaridad con los colegas. A quien informa de un acto ilícito se le puede considerar un mal jugador dentro del equipo y su carrera podría verse afectada negativamente. Esta presión podría verse reducida por la exigencia legal de informar de cualquier acto ilícito del que se haya tenido noticia, como en los EE.UU. O frecer protección estatutaria también puede ser de ayuda. Pero informar simplemente de un acto ilícito servirá de poco, a menos que se tenga que actuar por el hecho de que se ha informado y los funcionarios senior tengan obligación de solucionar el problema. Escribir una carta anónima puede ayudar a resolver algunos de los problemas mencionados anteriormente, pero muchos países prohíben iniciar una acción en base a una información anónima, pues esto podría conducir fácilmente a abusos.

Informar de los casos ilícitos es un asunto complejo y su éxito depende no sólo de las reglas formales, sino igual mente de la voluntad y capacidad de los funcionarios públicos senior. En cualquier caso, la cultura del silencio, que a menudo rodea las prácticas corruptas, debe cambiarse.

\section{Un gobierno más orientado al ciudadano}

Las reformas de la gestión pública en muchos países y en algunos en vías de desarrollo incluyen cambios hacia mejoras en la calidad de los servicios y la forma en la que el funcionariado trata al público. Esto puede ayudar a reducir la corrupción.

\section{Iniciativas en la calidad del servicio}

La escasa calidad del servicio es un rasgo frecuente de la prestación de servicios del sector público - el servicio puede no lle gar a los receptores deseados, puede no satisfacer las necesidades del receptor, puede suministrarsesobreuna basetemporal, y puede ser difícil de acceder - ya sea físicamente o porque los procedimientos burocráticos requieren un número considerable de formularios a cumplimentar, aprobaciones y esperas.

Cualquiera que sea la razón, la prestación deficiente de servicios refleja con frecuencia una cultura del funcionariado que se centra más en temas internos de cumplimiento de las normas 0 de promover su propio status y condiciones más que en prestar un servicio al público - ya sea ofrecer un tratamiento de salud, hacer una transferencia de pago o emitir una licencia o un permiso- . En esta cultura, no se considera que los ciudadanos o usuarios tengan un derecho específico a receibir determinados niveles de calidad del servicio, y los funcionarios tienen una discrecionalidad considerable respecto del nivel de calidad o servicio que prestan, si es que lo hay. Los funcionarios públicos, por tanto, pueden considerar que están haciendo un favor a los ciudadanos al proveerles del servicio. Los ciudadanos o usuarios pueden concluir que es necesario pagar a los funcionarios públicos para recibir un determinado servicio o nivel de calidad.

Como medio de mejorar la calidad de los servicios y luchar contra las prácticas corruptas, algunos gobiernos están adoptando «Cartas del Ciudadano» 0 Declaraciones de Estándares de Servicio, bajo los cuales se promulgan públicamente los derechos del ciudadano/usuario respecto de niveles 0 calidad concretos del servicio. Pero para que esta aproximación sea eficaz, es necesario 
educar tanto a los funcionarios públicos como a la sociedad civil y cambiar el comportamiento y las expectativas; el público puede no esperar altos niveles de servicio y puede no estar acostumbrado a quejarse, si no es así. C ambiar esta cultura puedellevar tiempo. Los mecanismos necesarios asociados son establecer objetivos de calidad de servicio claros e informar de la actuación real según estos objetivos, consultando con los ciudadanos sobre los aspectos clave de la prestación del servicio y desarrollando mecanismos efectivos de quejay satisfacción de las reclamaciones. También es importante que tal «enfoque en el cliente» no llegue a ser el de la «captura del cliente»; el gobierno más que los clientes tendría normalmente la última palabra sobre el nivel y calidad del servicio. Además, esto no es un derecho legal, sino más bien una declaración de lo que los clientes/usuarios esperan razonablemente recibir.

0 tra iniciativa importante sobre la calidad del servicio para reducir la corrupción puede ser la simplificación administrativa. Eliminando la documentación innecesaria o las aprobaciones para obtener servicios, sereducirá el número de «guardianes de la puerta», cada uno de los cuales puede exigir algún pago 0 «renta» antes de dar la aprobación.

Los cupones 0 comprobantes son otro mecanismo de prestación del servicio, que puede dar más poder a los ciudadanos/ usuarios. Estos ofrecen a los clientes/usuarios un derecho 0 autorización para utilizar servicios, tales como matricularse en una escuela o recibir un tratamiento médico. Los ciudadanos/usuarios simplemente presentan un cupón para obtener los servicios relevantes. El ciudadano/usuario, por tanto, puede elegir quéinstituciones educativas o sanitarias utilizar, lo cual puede aumentar la responsabilidad de los proveedores del servicio, pues no consiguen la financiación para continuar, a menos que puedan atraer usuarios. Los cupones se emplean en algunos países desarrollados, pero su aplicación en los países en vías de desarrollo requeriría un número adecuado de proveedores de servicios alternativos.

\section{Criterios para la toma de decisiones}

Las posibilidades de corrupción también se incrementan, a menos que se establezcan criterios claros de toma de decisiones sobre casos individuales ( $p$. e. la elegibilidad para recibir una pensión o una licencia) y que sean transparentes - conocidos y comprendidos tanto por los funcionarios públicos como por el público- . A este respecto, la transparencia en la toma de decisiones es un prerequisito importanteen la creación deun entorno quedesincentive las conductas ilícitas. Si no hay reglas claras, la discrecionalidad dada a los funcionarios públicos puede incentivar la corrupción. Además, debería haber alguna posibilidad de que los individuos pudieran plantear recursos contra tales decisiones administrativas, en un nivel inferior pidiendo una revisión interna de la decisión y en un nivel superior para impugnar una decisión ante un tribunal.
La complejidad administrativa innecesaria también puede contribuir a la corrupción. Si los criterios que tienen que ver con un servicio en concreto son demasiado complejos, pueden no ser entendidos, incrementando, por tanto, la discrecionalidad en la toma de decisiones de los funcionarios públicos y la posibilidad de recibir sobornos. La carencia de personal formado también hará aumentar este problema, por ejemplo, si la mayoría de los funcionarios públicos, incluidos sus supervisores, no entienden las reglas que se les exige aplicar, no se puede esperar que haya una toma de decisiones sensata y los ciudadanos pueden esperar tener que pagar sobornos para que se adopten las decisiones deseadas.

Sin embargo, también se debe reconocer que la mayor discrecionalidad de los funcionarios en decidir los casos específicos puede ser coherente con una aproximación más centrada en el cliente («una talla no vale a todo el mundo»). Pero tal discrecionalidad debe ejercerse según criterios claros, haciendo completamente responsables a todos los funcionarios por el ejercicio de esta discrecionalidad.

\section{Iniciativas de gestión del rendimiento}

En algunos países, se ha mejorado considerablemente la gestión del gobierno al centrarsela gestión del funcionariado más en los resultados. (Se emplean varios términos, incluido el de uresultados basados en la gestión», para describir estos avances). La gestión del gobierno puede ocuparse de temas relativos a la calidad del servicio, la eficiencia operativa y la eficacia de las organizaciones o programas a la hora de al canzar sus objetivos. Claramente, la corrupción, si implica el robo de dinero público o la prestación de un servicio público deficiente asociado a menudo con la corrupción, reducirá los niveles de gestión establecidos. Por ejemplo, pagar precios excesivos por ciertos artículos se pondrá de manifiesto a través de la medición de unos costes por unidad más elaborados de lo normal ${ }^{1}$. Los datos sugieren que la comparación puede ser un importante conductor del cambio.

Pero la cuestión clave de los sistemas de gestión del rendimiento son las consecuencias; si la demostrada mala gestión incentivará a hacer effuerzos para mejorar la gestión, incluida la lucha contra la corrupción. Pero de nuevo, esto requiere un entorno de gestión en el que haya iniciativas o presiones para llevarIo a cabo. Generalmente, la creación de este entorno llevará su tiempo. Los sistemas salariales basados en el rendimiento para jefes ejecutivos y otros funcionarios senior podrían introducirse como parte de éste. 
Todavía hay algunas advertencias asociadas con la aproximación de la gestión del rendimiento. Es importante que se midan los aspectos correctos de la gestión y que se establezcan los objetivos de gestión a los niveles adecuados. Si se miden las cosas inadecuadas o se establecen los objetivos a un nivel demasiado fácil, esto podría incentivar la corrupción donde la permanencia y el pago de un jefe ejecutivo dependen de la consecución de los objetivos de gestión. Además, para permitir que los gestores me joren el rendimiento, requieren la autonomía y flexibilidad adecuadas en la gestión presupuestaria y del personal. Conceder a los gestores autonomía operativa adicional, sin embargo, podría crear oportunidades para la corrupción. Es por tanto, importante que la concesión de autonomía o poder discrecional adicional vaya acompañada de responsabilidad adicional.

\section{Conclusión}

En muchos países en vías de desarrollo, las reformas de la administración pública no han logrado sus objetivos, no tanto por problemas de diseño sino a causa de los problemas con su implementación. Su aplicabilidad es la cuestión clave allí donde predominan las reglas informales. Con el tiempo, el sistema informal desarrolla sus propios valores y resiste el cambio.

La experiencia en las reformas del funcionariado sugiere que no se deberían separar artificialmente las tres disfunciones del estado ya conocidas -ineficacia, ineficiencia y corrupción. Un funcionariado que realiza una buena gestión no es corrupto, es eficiente y eficaz. Una disfunción puede crear otra.

En las anteriores reformas del funcionariado, los indicadores de gestión explícitos no se desarrollaron para efectuar mediciones de la reforma. Se necesita desarrollar indicadores anticorrupción e indicadores de eficacia para evaluar y mejorar los esfuerzos de reforma, además de desarrollar las herramientas de medición de su aplicación.

Los cambios en el contexto del gobierno invitan al funcionariado a adaptarse a los tiempos cambiantes, pero incluso en una economía condicionada por el mercado, el funcionariado jugaría un papel de liderazgo. Para jugar este papel, necesita cambiarse a sí mismo. Sólo una burocracia eficiente, eficaz y honesta puede acometer esta transición.

\section{Notas}

Artículo traducido por Gemma SÁn chez Rontomé.

$* N$. del T.: El término «C ivil Service» alude a todos los departamentos del gobierno que administran y gestionan los asuntos de un país, así como la gente que trabaja en ellos. No incluye a los miembros de las Fuerzas Armadas, el Parlamento, etc.

** Instituto del Banco M undial.
*** Instituto Internacional de la Administración Pública, París.

1 Los recientes Estudios de Prestación de Servicios del Banco M undial (World Bank Service D elivery) presentan una situación grave a este respecto, en la medida en que un alto porcentaje de funcionarios afirman que han sido obligados a pagar tales sobornos.

\section{Bibliografía}

Ban ERJI, Abhijit V. «A theory of misgovernance», IRIS Working Paper no. 126, 1994. Constitución de la República de U ganda.

C LAgUE, Christopher, ed.: Institutionsand Economic D evelopment, T heJ ohn H opkins University Press, Baltimore 1997.

EDI Evaluation Studies, ED I's Anti-corruption Initiatives in U ganda and Tanzania: A mid-term evaluation, 1998.

The Economist Intelligence Unit, Country Profile: Etiopía 1998-99.

EstaCHe, Antonio y D avid M ARTIM ORT. «Politics, Transaction costs and the D esign of Regulatory Institutions», Policy Research Working Paper 2073.

ICCA, Community Attitudes to Corruption and ICAC, Austria, 1996.

IM F, Policy Framework Papers, Etiopía, 1998/99-2000/01.

IM F, Policy Framework Papers, M alawi, 1998/99-2000/01.

IRIS, Governance and the Economy in Africa, Tools for analysis and Reform of Corruption.
K pundeh, Sahr y Petter LangSeTH, eds. U ganda Workshop of Parliamentarians on Good Governance for Sustainable Development, (Workshop Proceedings), Kampala, 1997.

LANGSETH, Petter. «Good Governance in Africa: A Case Study for U ganda», ED I Working Papers, 1997.

LAN GSETH, Petter y Rick StaPEN H URST. «N ational Integrity System C ountry Studies» (U ganda and Tanzania), EDI Working Papers, 1997.

LIEN ERT, Ian y Jitendra M ODI, «A D ecade of Civil Service Reforms in Sub-Saharan Africa», IM F, WP/97/179, 1997.

M ARKS, Christophe, «Decentralisation and the Potential for effective municipal governance in Senegal», IRIS Working Paper no. 133, 1976.

N un Berg, Barbara. «M anaging the Civil Service», World Bank D iscussion Papers, no. 204, 1995.

-----. Rethinking Civil Service Reform: An Agenda for smart government, 1997.

-----. «M anaging the Civil Service Reform», World Bank D iscussion Papers, no. 204. 
Nunberg, B. y John N ellis, «Civil Service Reform and the World Bank», World Bank Discussion Papers, N o. 161.

0 uat tara, AlassaneD . Viewsand Commentaries, Africa: An agenda for the $21^{ \pm}$century, IM F, 1999.

PUM A, OCDE, Public M anagement Reform and Economic and Social Development, 1998.

PUM A, OCDE, In Search of Results. Performance M anagement Practices, 1997.

PU M A, OCDE, Responsive Government; Service Quality Initiatives, 1996.

PU M A, OCDE, «Ethics in the Pulic Service: Current Issues and Practice», 0 ccasional Papers no. 14, 1996.

Stapenhurst, R. y Sahr J. Kpundeh, eds. Curbing Corruption: Towards a M odel for Building N ational Integrity, EDI D evelopment Studies, 1999.

Report of the Presidential Commission of Inquiry Against corruption inTanzania (el Informe Warioba), 1996.

«Recommendations for strengthening the anti-corruption programme», Uganda, PRSD Anti-Corruption Series no. 1, 1998.

«Towards a National Institutional Review for Ethiopia», Borrador para una discusión interna, 1999.
The United Republic of Tanzania, The National Integrity System in Tanzania (Workshop Proceedings), 1995.

Van Rijckeghem, Caroliney B. Weder, «Corruption and the Rate of Temptation: Do low wages in Civil Service cause corruption», IM F Working Paper, 97/73.

W ORLD BAnk. «Civil Service Reform: A Review of the World Bank Assistance», OED Report no. 1921, 1999.

-----. «Governance and Structural Adjustment in Ghana», World Bank Working Paper 1995.

.G overnance Approach to Civil Service Reform in Africa», World Bank Technical Papers, no. 225, 1997

-----. «G overnment Employment and Pay: A global and regional perspective», World Bank Policy Research Working Paper 1771, 1997.

. «An International statistical survey of government employment and wages», WBPRWP 1806, 1997.

M alawi: Support to the Government of M alawi's anti-corruption programme, PRSPU (Africa Región), Nov. 1998.

-----. Corruption and Political Finance in Africa, PRSPU, Africa Región, 1998.

-----. Support to the Government of Tanzania's Anti-Corruption Programme, PRSPU, 1998. 